\title{
Dupilumab in persistent asthma with elevated eosinophil levels
}

Monoclonal antibodies are an effective treatment for a number of inflammatory autoimmune conditions, particularly those refractory to first-line medications. In asthma, airway inflammation may be associated with Th2 cell overactivity and raised eosinophils in up to $50 \%$ of cases. Th2 activation leads to the release of interleukin (IL)-13 and IL-4, which mediate their effects via receptors containing the IL- $4 \alpha$ subunit. Dupilumab is an IL- $4 \alpha$ subunit inhibitor aiming to block both signalling pathways and reduce airway inflammation.

The authors conducted a randomised, double-blind, placebo-controlled study to evaluate the effectiveness and safety of dupilumab in the treatment of persistent moderate-to-severe asthma. They recruited 104 patients, aged 18-65, who had an elevated blood or sputum eosinophil count and symptoms that remained poorly controlled despite medium-dose to high-dose inhaled corticosteroid (ICS) and long-acting bronchodilators (LABAs). Patients received once-weekly subcutaneous injections of dupilumab or placebo for 12 weeks or until they suffered an asthma exacerbation. LABAs were discontinued at week 4, and ICSs tapered from week 6 to stop by week 9 .

The primary outcome was an asthma exacerbation requiring systemic steroids or hospital attendance. This was seen in $6 \%$ of those receiving dupilumab and $44 \%$ of those receiving placebo. In terms of secondary outcomes, dupilumab patients showed significant improvements in $\mathrm{FEV}_{1}$, morning peak respiratory flow rate, asthma symptoms, nocturnal awakening, salbutamol use and Th2-associated inflammatory markers.

These promising results warrant further investigation over a longer time frame. Adverse effects, notably injection-site reactions, nasopharyngitis, nausea and headache, were reported more frequently with dupilumab; hence, further studies are required to determine its longterm safety and side-effect profile, something that may ultimately affect patient compliance.

- Wenzel S, Ford L, Pearlman D, et al. Dupilumab in persistent asthma with elevated eosinophil levels. N Engl J Med 2013;368:2455-66.

Ruksha Bhadresha

Correspondence to Dr Ruksha Bhadresha, FY2 East Sussex Healthcare NHS Trust, Conquest Hospital, The Ridge, St Leonards-on-Sea, East Sussex TN37 7RD, UK; ruksha.bhadresha@nhs.net

To cite Bhadresha R. Thorax 2014;69:708.

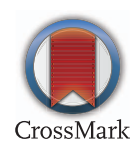

Published Online First 18 September 2013

Thorax 2014;69:708. doi:10.1136/thoraxjnl-2013-204437 